\title{
Recent results from the NA62 experiment
}

\author{
Elisa Minucci ${ }^{a, 1,2}$ \\ ${ }^{a}$ CERN,Geneva, Switzerland \\ E-mail: elisa.minucci@cern.ch
}

The NA62 experiment at the CERN SPS, designed to measure the branching ratio of the ultra-rare $K^{+} \rightarrow \pi^{+} v \bar{v}$ decay using a decay-in-flight technique, collected data from 2016 to 2018 . The analysis of the full 2018 data set is presented here, corresponding to a total number of kaon decays of about $2.62 \times 10^{12}$, the largest data set so far collected. The combination with the 2016-2017 data set is also reported. In addition the latest results from other searches are briefly presented.

\section{BEAUTY2020}

21-24 September 2020

Kashiwa, Japan (online)

${ }^{1}$ On behalf of the NA62 Collaboration: R. Aliberti, F. Ambrosino, R. Ammendola, B. Angelucci, A. Antonelli, G. Anzivino, R. Arcidiacono, T. Bache, A. Baeva, D. Baigarashev, M. Barbanera, J. Bernhard, A. Biagioni, L. Bician, C. Biino, A. Bizzeti, T. Blazek, B. Bloch-Devaux, V. Bonaiuto, M. Boretto, M. Bragadireanu, D. Britton, F. Brizioli, M.B. Brunetti, D. Bryman, F. Bucci, T. Capussela, J. Carmignani, A. Ceccucci, P. Cenci, V. Cerny, C. Cerri, B. Checcucci, A. Conovaloff, P. Cooper, E. Cortina Gil, M. Corvino, F. Costantini, A. Cotta Ramusino, D. Coward, G. D’Agostini, J. Dainton, P. Dalpiaz, H. Danielsson, N. De Simone, D. Di Filippo, L. Di Lella, N. Doble, B. Dobrich, F. Duval, V. Duk, D. Emelyanov, J. Engelfried, T. Enik, N. Estrada-Tristan, V. Falaleev, R. Fantechi, V. Fascianelli, L. Federici, S. Fedotov, A. Filippi, M. Fiorini, J. Fry, J. Fu, A. Fucci, L. Fulton, E. Gamberini, L. Gatignon, G. Georgiev, S. Ghinescu, A. Gianoli, M. Giorgi, S. Giudici, F. Gonnella, E. Goudzovski, C. Graham, R. Guida, E. Gushchin, F. Hahn, H. Heath, J. Henshaw, E.B. Holzer, T. Husek, O. Hutanu, D. Hutchcroft, L. Iacobuzio, E. Iacopini, E. Imbergamo, B. Jenninger, J. Jerhot, R.W. Jones, K. Kampf, V. Kekelidze, S. Kholodenko, G. Khoriauli, A. Khotyantsev, A. Kleimenova, A. Korotkova, M. Koval, V. Kozhuharov, Z. Kucerova, Y. Kudenko, J. Kunze, V. Kurochka, V. Kurshetsov,G. Lanfranchi, G. Lamanna, E. Lari, G. Latino, P. Laycock, C. Lazzeroni, M. Lenti, G. Lehmann Miotto, E. Leonardi, P. Lichard, L. Litov, R. Lollini, D. Lomidze, A. Lonardo, P. Lubrano, M. Lupi, N. Lurkin, D. Madigozhin, I. Mannelli, A. Mapelli, F. Marchetto, R. Marchevski, S. Martellotti, P. Massarotti, K. Massri, E. Maurice, M. Medvedeva, A. Mefodev, E. Menichetti, E. Migliore, E. Minucci, M. Mirra, M. Misheva, N. Molokanova, M. Moulson, S. Movchan, M. Napolitano, I. Neri, F. Newson, A. Norton, M. Noy, T. Numao, V. Obraztsov, A. Ostankov,S. Padolski, R. Page, V. Palladino, A. Parenti, C. Parkinson, E. Pedreschi, M. Pepe, M. Perrin-Terrin, L. Peruzzo, P. Petrov, Y. Petrov, F. Petrucci, R. Piandani, M. Piccini, J. Pinzino, I. Polenkevich, L. Pontisso, Yu. Potrebenikov, D. Protopopescu, M. Raggi, A. Romano, P. Rubin, G. Ruggiero, V. Ryjov, A. Salamon, C. Santoni, G. Saracino, F. Sargeni, S. Schuchmann, V. Semenov, A. Sergi, A. Shaikhiev, S. Shkarovskiy, D. Soldi, V. Sugonyaev, M. Sozzi, T. Spadaro, F. Spinella, A. Sturgess, J. Swallow, S. Trilov, P. Valente, B. Velghe, S. Venditti, P. Vicini, R. Volpe, M. Vormstein, H. Wahl, R. Wanke, B. Wrona, O. Yushchenko, M. Zamkovsky, A. Zinchenko.

${ }^{2}$ Supported by ERC-STG802836 (AxScale) 


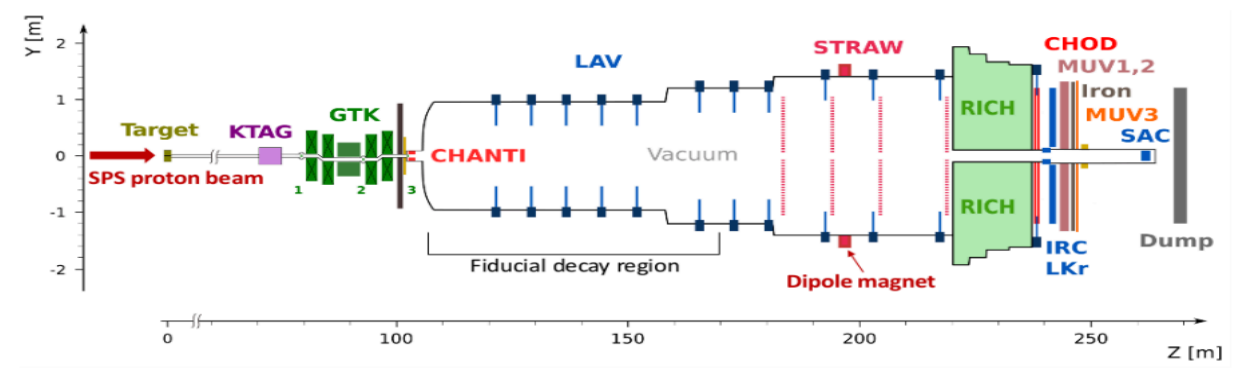

Figure 1: Top view of the NA62 beamline and detector.

\section{Introduction}

The ultra-rare $K^{+} \rightarrow \pi^{+} v \bar{v}$ decay is a flavour changing neutral current process, proceeding through box and electroweak penguin diagrams. It is theoretically well predicted and sensitive to new physics beyond the Standard Model (SM), probing high mass scales [1, 2]. The SM branching ratio is:

$$
\operatorname{Br}\left(K^{+} \rightarrow \pi^{+} v \bar{v}\right)_{S M}=(8.4 \pm 1.0) \times 10^{-11}
$$

where the main source of uncertainty originates from the uncertainties on the CKM matrix elements [3]. Previous experimental result was based on seven signal events collected by the BNL-AGSE787(E949) experiments, that measured a branching ratio of $\operatorname{Br}\left(K^{+} \rightarrow \pi^{+} v \bar{v}\right)=\left(17.3_{-10.5}^{+11.5}\right) \times 10^{11}$ using the stopped kaons technique [4].

\section{The NA62 detector}

The NA62 beam line and detector are described in detail in [5] and shown in Figure 1. The NA62 beam is produced by the interaction of a $400 \mathrm{GeV} / c$ proton beam, coming from the CERN super proton syncrotron (SPS), with a beryllium target. It is composed of positive charged particles of which $6 \%$ are kaons and it has a central momentum of $75 \mathrm{GeV} / \mathrm{c}$ with a momentum bite of $\Delta p / p=1 \%$. The kaons in the beam are identified by a Cherenkov counter detector, KTAG. Before entering the vacuum region downstream, the kaon momentum and direction are measured by the Gigatracker (GTK), composed of three silicon pixel stations of $6 \times 3 \mathrm{~cm}^{2}$ surface area exposed to the full beam rate. Inelastic interactions in the last GTK station (GTK3) are vetoed by the CHANTI, composed of six stations of plastic scintillator bars. Downstream, a magnetic spectrometer, made of four STRAW chambers and a dipole magnet measures the momentum and direction of charged particles coming from kaon decays. A ring-imaging Cherenkov detector $(\mathrm{RICH})$ is used to separate $\pi^{+}, \mu^{+}$and $e^{+}$. The time of charged particles is measured both with the RICH and with an array of scintillators (CHOD) located downstream of the RICH. Two hadronic calorimeters (MUV1 and MUV2) and a fast scintillator array (MUV3) provide further separation between $\pi^{+}$and $\mu^{+}$. Photons are detected by an hermetic photon veto system covering angles up to $50 \mathrm{mrad}$ from the beam axis, composed of twelve annular lead glass detectors (LAV1-12), two lead/scintillator sampling calorimeters (IRC, SAC) close to the beam axis and a liquid krypton electromagnetic calorimeter $(\mathrm{LKr})$. 

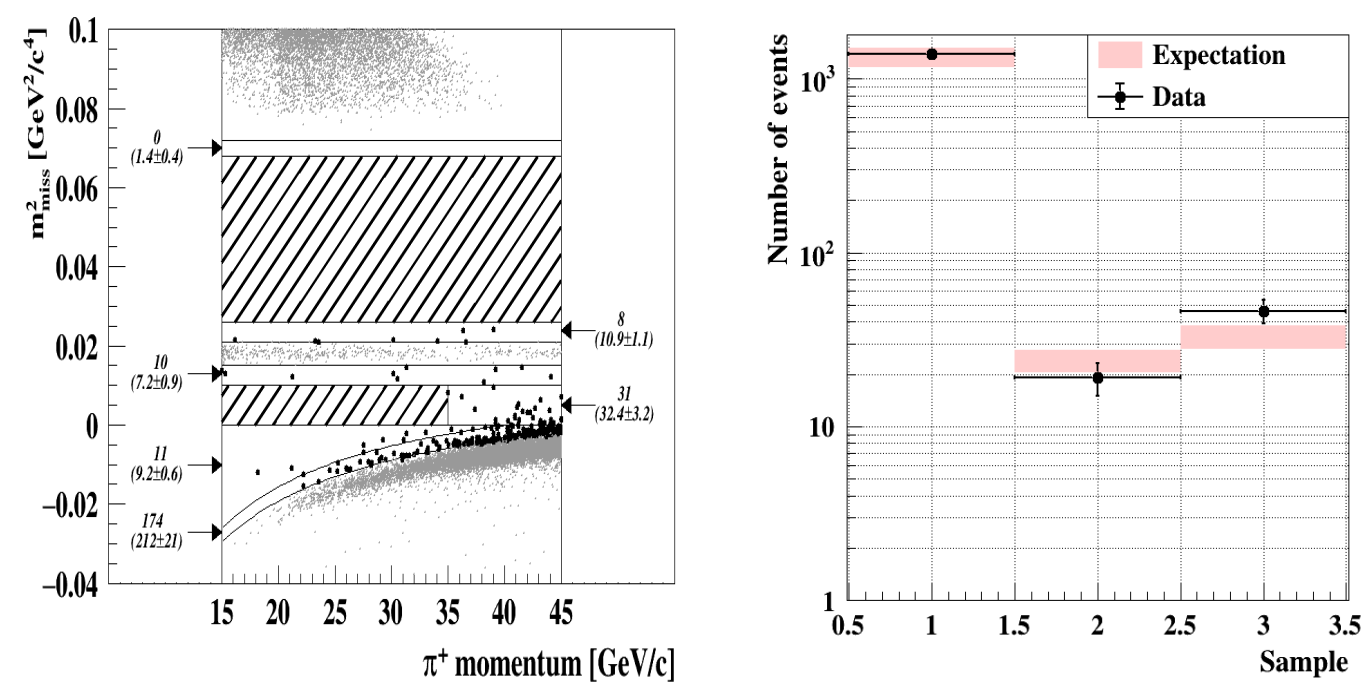

Figure 2: Left: Reconstructed $m_{\text {miss }}^{2}$ as a function of $\pi^{+}$momentum after the complete signal selection. In the plot the background regions are depicted by the small grey dots. The background validation regions, depicted by the solid black dots, are kept blind until the analysis is complete. The numbers next to those regions are the expected number of background events (in brackets) and the observed number. The signal regions, indicated by the hatched rectangles, are kept blind until the completion of the analysis. Right: Comparison between expected and observed number of events for three upstream background validation samples. Those samples, enriched of upstream background events, are selected by inverting some of the selection criteria used in the analysis.

\section{The $K^{+} \rightarrow \pi^{+} v \bar{v}$ analysis}

The experimental signature of the signal consists of a $K^{+}$with 4-momentum $p_{K}$ in the initial state and a $\pi^{+}$with 4-momentum $p_{\pi}$ in the final state plus missing energy from the neutrinos. The kinematic variable used to distinguish the signal from other $K^{+}-$decay backgrounds and to define the signal regions is the squared missing mass $m_{\text {miss }}^{2}=\left(p_{K}-p_{\pi}\right)^{2}$.

Events with a single-track decay topology are selected using the downstream detectors STRAW, CHOD and RICH. The downstream track reconstructed with the STRAW must be associated to a signal in the CHOD and to a reconstructed ring in the $\mathrm{RICH}$, which defines the track time with $100 \mathrm{ps}$ precision. The selected downstream track is associated to an in-time $K^{+}$in the KTAG detector. A $K^{+}$is reconstructed in the GTK. A kaon decay vertex is reconstructed and a cut is applied to be inside a $50 \mathrm{~m}$ long fiducial region, starting $10 \mathrm{~m}$ downstream of GTK3. The downstream track is identified as a $\pi^{+}$using two complementary methods: a multivariate analysis with Boosted Decision Trees (BDT) using energy deposition, energy sharing and shower shape profiles in the LKr and MUV1/2, as well as signals from MUV3; a cut-based approach using the particle mass reconstructed by the RICH detector and a track-driven likelihood discriminant for $\pi^{+} / \mu^{+} / e^{+}$separation. The algorithm for particle identification has an efficiency for identifying $\pi^{+}$of $64 \%$ and a muon mis-identification probability of $O\left(10^{-8}\right)$. Additionally a photon and multi-charged particle rejection is applied based on information in the photon veto detectors and the CHOD. The photon veto conditions provide a $\pi^{0}$ suppression of $O\left(10^{-8}\right)$. The $m_{m i s s}^{2}$ distribution after the complete signal selection is shown in 
Figure 2 (left).

The the 2018 data set is divided in two periods: S1 and S2, before and after the installation of a new collimator, corresponding to $20 \%$ and $80 \%$ of the full statistics respectively. The analysis of the S1 sample follows the analysis of the 2017 data, described in [6], with small optimizations, while major improvements have been applied in the analysis of the $\mathrm{S} 2$ sample. The $K^{+} \rightarrow \pi^{+} v \bar{v}$ is normalized to the $K^{+} \rightarrow \pi^{+} \pi^{0}$ decay. This allows cancellation of systematic effects related to both $K^{+}$and $\pi^{+}$.

The number of expected SM signal events is derived from

$$
N_{\text {exp }}=N_{\pi \pi} \varepsilon_{t r i g} \varepsilon_{R V} \frac{A_{\pi v v} B r(\pi v v)}{A_{\pi \pi} B r(\pi \pi)}=\left(7.58 \pm 0.40_{\text {syst }} \pm 0.75_{\text {ext }}\right)
$$

where $N_{\pi \pi}$ is the number of $K^{+} \rightarrow \pi^{+} \pi^{0}$ decays selected from a minimum bias sample, $A_{\pi v v}$ and $A_{\pi \pi}$ are the acceptance of the signal and normalization channels derived from Monte Carlo simulations, $\varepsilon_{\text {trig }}$ is the trigger efficiency and $\varepsilon_{R V}$ is random veto efficiency, derived from data, induced by the photon and multi-charged particle rejection procedure. $\operatorname{Br}(\pi v v)$ and $\operatorname{Br}(\pi \pi)$ are the SM branching ratio of the signal and normalization channels, respectively. The quoted systematic uncertainty receives the main contributions from: trigger efficiency, random veto losses and simulation of the $\pi^{+}$losses due to interactions in the detector material upstream of the hodoscopes. The external uncertainty is due to the theoretical knowledge of the $K^{+} \rightarrow \pi^{+} v \bar{v}$ branching ratio.

The Single Event Sensitivity (S.E.S) for the 2018 data set is:

$$
\text { S.E.S }=\frac{B r(\pi v v)}{N_{\text {exp }}}=\left(1.11 \pm 0.07_{\text {syst }}\right) \times 10^{-11} .
$$

The expected number of background events in the signal region is $N_{b k g}=\left(5.28_{-0.74}^{+0.99}\right)$. The background is composed of $K^{+}$decays in the fiducial region (1.98 \pm 0.15 events) and upstream background $\left(3.30_{-0.73}^{+0.98}\right.$ events) due to upstream $K^{+}$decays forming a fake vertex with a beam particle inside the fiducial region. The background from the more abundant kaon decays, $K^{+} \rightarrow \pi^{+} \pi^{0}(\gamma)$, $K^{+} \rightarrow \mu^{+} v(\gamma)$ and $K^{+} \rightarrow \pi^{+} \pi^{+} \pi^{-}$, in the signal region is validated in dedicated background validation regions (see Figure 2 (left)). The upstream and $K^{+} \rightarrow \pi^{+} \pi^{-} e^{+} v$ background predictions are validated using separate samples obtained by inverting some of the criteria used for the $K^{+} \rightarrow$ $\pi^{+} v \bar{v}$ selection (see Figure 2 (right)).

\section{Result}

After unblinding the two signal regions 17 events have been observed in the 2018 data set, distributed as shown is Figure 3 (left). In total, adding the result of the $K^{+} \rightarrow \pi^{+} v \bar{v}$ analysis performed on the 2016 [7], 2017 [6] and 2018 data, NA62 has observed 20 candidate events. The final branching ratio is obtained performing a maximum likelihood fit using the signal and background expectation in 9 categories illustrated in Figure 3 (right), including also the 2017 and 2016 results. The resulting branching ratio is

$$
\operatorname{Br}\left(K^{+} \rightarrow \pi^{+} v \bar{v}\right)=\left(11.0_{-3.5}^{+4.0} \pm 0.3\right) \times 10^{-11}
$$

The first uncertainty is statistical, dominated by the Poissonian fluctuation of the expected background, and the second is systematic. The result is compatible with the SM value within one 

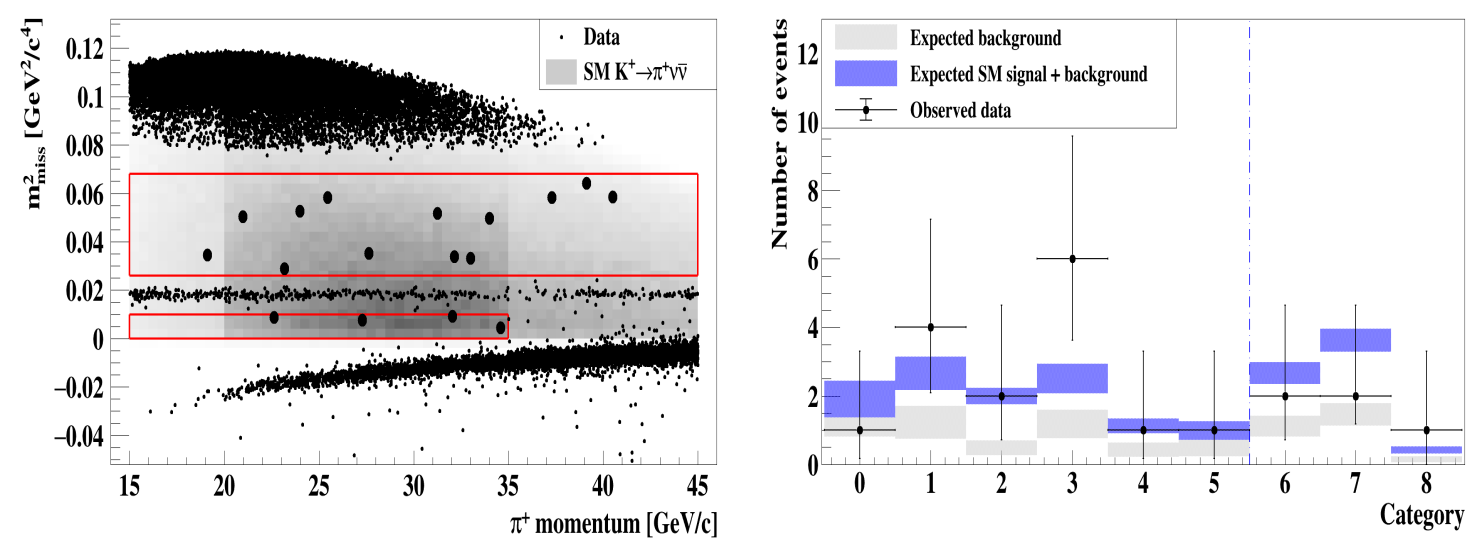

Figure 3: Left: Reconstructed $m_{m i s s}^{2}$ as a function of $\pi^{+}$momentum after the unblinding of the signal regions (two red boxes). The grey area corresponds to the expected distribution of $K^{+} \rightarrow \pi^{+} v \bar{v}$ MC events. Right: Comparison between the expected and observed number of events in the different categories used in the maximum likelihood fit to extract the $K^{+} \rightarrow \pi^{+} v \bar{v}$ branching ratio. Categories 0 to 5 correspond to the six $5 \mathrm{GeV} / c$-wide momentum bins of the $2018 \mathrm{~S} 2$ sample. Categories 6,7 and 8 correspond to the $2018 \mathrm{~S} 1$, 2017 and 2016 samples, respectively.

standard deviation and in a background-only hypothesis the signal significance is $3.5 \sigma$, estimated using the CLs method. This is the most precise measurement of the $K^{+} \rightarrow \pi^{+} v \bar{v}$ branching ratio.

\section{Other analyses}

In addition to the main analysis, NA62 performs other searches, collecting data in parallel with the $K^{+} \rightarrow \pi^{+} v \bar{v}$ data taking.

Test of Lepton Flavour Universality (LFU) Tests of LFU can be performed measuring the $K^{+} \rightarrow \pi^{+} \mu^{+} \mu^{-}$form factor parameters which LFU predicts independently of the lepton flavour [8]. The results obtained from the analysis of the $K^{+} \rightarrow \pi^{+} \mu^{+} \mu^{-}$decay on the 2017-2018 data set reported in Figure 4 are compatible with previous measurements in both muon and electron modes and thus no deviation from LFU is observed.

Lepton flavour and lepton number violation searches Lepton number (LN) and Lepton Flavour number (LF) can occur in models beyond the SM. Searches of LN and LF violation are powerful probes to access high mass scales. From the analysis of the full 2017-2018 data set, new limits have been set in semi-leptonic $K^{+} \mathrm{LN}$ and LF violating decays, improving on previous limits [9] by one order of magnitude, reaching sensitivity of $O\left(10^{-11}\right): \mathcal{B}\left(K^{+} \rightarrow \pi^{-} \mu^{+} e^{+}\right)<4.2 \times 10^{-11}$, $\mathcal{B}\left(K^{+} \rightarrow \pi^{+} \mu^{-} e^{+}\right)<6.6 \times 10^{-11}$ at $90 \%$ C.L.

Heavy neutral lepton The NA62 experiment at CERN collected large samples of kaon decays, allowing to search for heavy neutrino production in $K^{+} \rightarrow l^{+} v_{h}$ decays $(l=\mu, e)$. The analysis of the electron channel has been performed on the full data set reaching limits on the $\left|U_{e 4}\right|$ parameter of $O\left(10^{-9}\right)$ [10]. The analysis on the muon channel has been done on $30 \%$ of the full statistics reaching sensitivity on the $\left|U_{\mu 4}\right|$ parameter of $O\left(10^{-8}\right)$. 

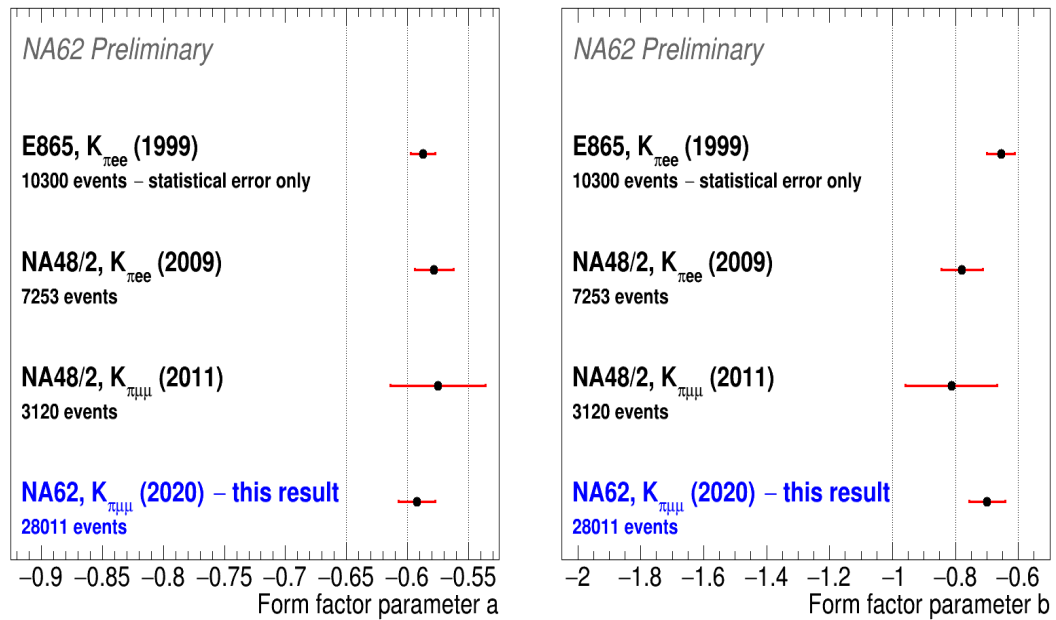

Figure 4: Form factor parameters $a$ and $b$ : the latest NA62 measurement in the muon mode and previous measurements in both muon and electron modes.

\section{Summary}

The NA62 experiment took data from 2016 to 2018 , collecting $\sim 2.2 \times 10^{18}$ protons on target. Combining the results obtained from the whole data set the most precise measurement of the $K^{+} \rightarrow \pi^{+} v \bar{v}$ branching ratio has been set: $\operatorname{Br}\left(K^{+} \rightarrow \pi^{+} v \bar{v}\right)=\left(11.0_{-3.5}^{+4.0} \pm 0.3\right) \times 10^{-11}$. In addition NA62 performed searches of rare and forbidden kaon decays and exotic particles. NA62 will resume data taking in 2021 with an upgraded detector allowing for an improved signal sensitivity.

\section{References}

[1] A. J. Buras, D. Buttazzo, R. Knegjens, JHEP 1511, 166 (2015)

[2] M. Bordone, D. Buttazzo, G. Isidori, J. Monnard, Eur. Phys. J. C77, 618 (2017)

[3] A. J. Buras, D. Buttazzo, K. Girrbach-Noe, R. Knegjens, JHEP 11, 033 (2015)

[4] A. V. Artamonov et al., Phys. Rev. D79, 092004 (2009)

[5] The NA62 Collaboration, JINST 12, P05025 (2017)

[6] The NA62 Collaboration, JHEP 11, 042 (2020)

[7] The NA62 Collaboration, Phys. Lett. B791, 156-166 (2019)

[8] G. D’Ambrosio et al. JHEP 08, 004 (1998)

[9] R. Appel et al., Phys. Rev. Lett. 85, 2877 (2000)

[10] The NA62 Collaboration, Phys. Lett. B807, 135599 (2020) 